\title{
RESEARCH
}

Open Access

\section{CD36-mediated uptake of myelin debris by macrophages and microglia reduces neuroinflammation}

Elien Grajchen ${ }^{1}$, Elien Wouters ${ }^{1}$, Britt van de Haterd ${ }^{1}$, Mansour Haidar ${ }^{1}$, Kévin Hardonnière², Tess Dierckx Jana Van Broeckhoven ${ }^{1}$, Celine Erens ${ }^{1}$, Sven Hendrix ${ }^{1}$, Saadia Kerdine-Römer ${ }^{2}$, Jerome J. A. Hendriks ${ }^{1+}$ and Jeroen F. J. Bogie ${ }^{1 *+}$ (D)

\begin{abstract}
Background: The presence of foamy macrophages and microglia containing intracellular myelin remnants is a pathological hallmark of neurodegenerative disorders such as multiple sclerosis (MS). Despite the importance of myelin internalization in affecting both central nervous system repair and neuroinflammation, the receptors involved in myelin clearance and their impact on the phagocyte phenotype and lesion progression remain to be clarified.

Methods: Flow cytometry, quantitative PCR, and immunohistochemistry were used to define the mRNA and protein abundance of CD36 in myelin-containing phagocytes. The impact of CD36 and nuclear factor erythroid 2related factor 2 (NRF2) on the phagocytic and inflammatory features of macrophages and microglia was assessed using a pharmacological CD36 inhibitor (sulfo-N-succinimidyl oleate) and $\mathrm{Nrf}^{-/-}$bone marrow-derived macrophages. Finally, the experimental autoimmune encephalomyelitis (EAE) model was used to establish the impact of CD36 inhibition on neuroinflammation and myelin phagocytosis in vivo.

Results: Here, we show that the fatty acid translocase CD36 is required for the uptake of myelin debris by macrophages and microglia, and that myelin internalization increased CD36 expression through NRF2. Pharmacological inhibition of CD36 promoted the inflammatory properties of myelin-containing macrophages and microglia in vitro, which was paralleled by a reduced activity of the anti-inflammatory lipid-sensing liver $X$ receptors and peroxisome proliferatoractivated receptors. By using the EAE model, we provide evidence that CD36 is essential for myelin debris clearance in vivo. Importantly, CD36 inhibition markedly increased the neuroinflammatory burden and disease severity in the EAE model.
\end{abstract}

Conclusion: Altogether, we show for the first time that CD36 is crucial for clearing myelin debris and suppressing neuroinflammation in demyelinating disorders such as MS.

Keywords: Macrophages, Microglia, Myelin, CD36, Neuroinflammation, Fatty acid, Multiple sclerosis

\footnotetext{
* Correspondence: Jeroen.bogie@uhasselt.be

${ }^{\dagger}$ Jerome J. A. Hendriks and Jeroen F. J. Bogie contributed equally to this work.

${ }^{1}$ Department of Immunology and Infection, Biomedical Research Institute, Hasselt University, Diepenbeek, Belgium

Full list of author information is available at the end of the article
} 


\section{Introduction}

The internalization of myelin by phagocytes and the subsequent formation of foamy phagocytes is a pathological hallmark of neurodegenerative disorders [1, 2]. In multiple sclerosis (MS), the presence of foamy phagocytes is even used as an index of MS lesion activity [3]. While myelin internalization was initially regarded to underlie demyelination in neurodegenerative disorders, increasing evidence indicates that clearance of myelin debris by phagocytes is also necessary for central nervous system (CNS) repair $[4,5]$. Aside from directly affecting demyelination and remyelination, ample evidence indicates that myelin internalization controls the functional properties of phagocytes as well. For instance, we and others showed that uptake of myelin skews phagocytes towards an immunosuppressive and neurotrophic phenotype [6-8]. This protective phenotype is closely associated with the activation of lipid-responsive signaling pathways, such as the liver X receptor (LXR) and peroxisome proliferator-activated receptor (PPAR) signaling pathways [9-11]. More recent findings indicate that sustained uptake and intracellular accumulation of myelin-derived cholesterol impairs the metabolic and protective features of foamy phagocytes, and instead skews these cells towards an inflammatory, disease-promoting phenotype [12, 13]. Collectively, these findings indicate that myelin internalization is an essential process in the regulation of phagocyte function and disease pathology in demyelinating disorders.

To date, numerous receptors are reported to drive the internalization of myelin. Early studies defined that Fc, complement, and scavenger receptors control myelin phagocytosis [1]. More recent studies indicate that mer tyrosine kinase, low-density lipoprotein receptor-related protein 1 , and collectin placenta 1 (CLP1) are also functional regulators of myelin uptake by macrophages and microglia [14-16]. Of interest, many of the abovementioned receptors also regulate the binding and uptake of modified LDL [17-19], A $\beta$ peptides [20-23], and apoptotic cells [24-26]. However, despite being a key receptor in the internalization of these ligands, it remains unclear whether the scavenger receptor CD36 is involved in the phagocytosis of myelin. CD36 is a member of the class B scavenger receptor family and is expressed on various cell types, including monocytes, macrophages, endothelial cells, and adipocytes [27]. Cd36 deficiency in mice and humans decreases fatty acid uptake in muscle and adipose tissues [28, 29], providing evidence that CD36 acts as a fatty acid translocase. In atherosclerosis, $C d 36$ deficiency in macrophages protects mice against lesion development [30-32], consistent with the detrimental role of oxLDL uptake and the formation of foamy macrophages in atherosclerotic lesion progression. Noteworthy, CD36 internalizes oxLDL by a mechanism dependent on the binding of fatty acids [33], suggesting that the presence of fatty acids is important for recognition by CD36. On a transcriptional level, PPAR $\gamma$ and nuclear factor erythroid 2-related factor 2 (NRF2) are amongst the transcription factors that regulate CD36 abundance [34, 35]. Altogether, these studies indicate that CD36 is a phagocytic receptor that plays a vital role in the uptake of fatty acid-containing substrates.

Given the abundance of fatty acids in myelin, we defined in this study if CD36 controls the uptake of myelin by macrophages and microglia. We show that CD36 protein levels are significantly elevated in myelin-containing phagocytes in vitro and in vivo. CD36 protein level was primarily regulated through the NRF2 signaling pathway. Pharmacological inhibition of CD36 reduced the uptake of myelin debris and promoted neuroinflammation in vitro and in vivo. Our findings highlight the importance of functional CD36 in clearing myelin debris and suppressing neuroinflammation in demyelinating CNS disorders such as MS.

\section{Materials and methods \\ Antibodies and chemical reagents}

The following antibodies were used for flow cytometry and immunofluorescent/immunohistochemical stainings: anti-CD36 (1:100, cat. \#ab80080, Abcam), anti-IBA1 (1: 500, cat. \#019-19741, Fujifilm), anti-CD68 (1:100, cat. \#14-0688, Invitrogen), anti-dMBP (1:2000, cat. \#ab5864, Sigma-Aldrich), Alexa Fluor 700-labeled anti-CD45 (1: 200, cat. \#103128, BioLegend), Zombie NIR (1:1000, cat. \#423106, BioLegend), anti-NOS2 (1:100, cat. \#ab15323, Abcam), and anti-F4/80 (1:100, cat. \#MCA497G, BioRad). Appropriate secondary antibodies were purchased from Invitrogen. BODIPY (493/503) was used to fluorescently label lipid droplets $(2 \mu \mathrm{M}$, cat. \#D3922, Invitrogen). 7-Aminoactinomycin D (7AAD, $0.5 \mu \mathrm{g} / \mathrm{ml}$, Thermo Fisher Scientific) was used to assess cellular viability. Sulfo-N-succinimidyl oleate (SSO, $100 \mu \mathrm{M}$, cat. \#11211, Cayman Chemical) and GW9662 $(10 \mu \mathrm{M}$, cat. \#M6191, Sigma-Aldrich) were used to inhibit CD36 and PPAR $\gamma$, respectively. Lipopolysaccharide (LPS, $100 \mathrm{ng} / \mathrm{ml}$, SigmaAldrich) was used to stimulate cells for inflammatory phenotyping.

\section{Mice}

Female wild-type (wt) C57BL/6 J mice were purchased from Envigo. $\mathrm{Nrf}^{-1-}$ mice were provided by the RIKEN BRC according to an MTA to Prof S. Kerdine-Römer $[35,36]$. Mice were housed in a pathogen-free facility and handled in accordance with the principles and procedures outlined in Council Directive 86/609/EEC. Genotyping was performed by PCR using genomic DNA that was isolated from tail snips as described previously $[35,36]$. Mice were maintained on a $12 \mathrm{~h}$ light/dark 
cycle with free access to water and a standard chow diet (Ssniff). All studies were conducted in accordance with the institutional guidelines and approved by the Ethical Committee for Animal Experiments of Hasselt University.

Experimental autoimmune encephalomyelitis (EAE) model Twelve-week-old female wt C57BL/6 mice were immunized subcutaneously with $200 \mu \mathrm{g}$ of recombinant human myelin oligodendrocyte glycoprotein $\mathrm{MOG}_{35-55}$, emulsified in $100 \mu \mathrm{l}$ complete Freund's adjuvant supplemented with $4 \mathrm{mg} / \mathrm{ml}$ of Mycobacterium tuberculosis (H37RA strain) according to manufacturer's guidelines (Hooke Laboratories). Within $2 \mathrm{~h}$ and after 22-26h, mice were intraperitoneally injected with $25 \mathrm{ng}$ pertussis toxin. Immunized mice were weighed and scored daily following a five-point standardized rating of clinical symptoms, 0 , no signs; 0.5 , distal tail paralysis; 1 , loss of tail tonus; 2, flaccid tail; 3 , hind limb paresis; 4, hind limb paralysis; and 5, death. Starting from day 9 postimmunization or when a disease score of 0.5 or higher was obtained, animals were injected intraperitoneally with SSO $(30 \mathrm{mg} / \mathrm{kg})$ or vehicle on a daily basis. Mice were sacrificed at day 22 post-immunization or at 11 days post-disease onset.

\section{Cell isolation and culture}

Bone marrow-derived macrophages (BMDMs) were obtained as described previously [14]. Briefly, femoral and tibial bone marrow cell suspensions from 12-week-old wt and $\mathrm{Nrf}^{-/-} \mathrm{C} 57 \mathrm{Bl} / 6$ mice were cultured in $10 \mathrm{~cm}$ plates at a concentration of $10 \times 10^{6}$ cells/plate and differentiated in RPMI 1640 medium (Lonza) supplemented with $10 \%$ fetal calf serum (FCS, Gibco), $50 \mathrm{U} / \mathrm{ml}$ penicillin (Invitrogen), $50 \mathrm{U} / \mathrm{ml}$ streptomycin (Invitrogen), and 15\% L929-conditioned medium (LCM). After differentiation, cells were cultured $\left(0.5 \times 10^{6}\right.$ cells $\left./ \mathrm{ml}\right)$ in RPMI 1640 supplemented with 10\% FCS, $50 \mathrm{U} / \mathrm{ml}$ penicillin, $50 \mathrm{U} / \mathrm{ml}$ streptomycin, and 5\% LCM. Primary microglia cell cultures were prepared from brains of postnatal P1-3 C57BL/6 pups. Carefully, the brain stem, choroid plexus, and meninges were removed before brains were enzymatically digested for $15 \mathrm{~min}$ with $1 \mathrm{x}$ trypsin (Gibco) at $37^{\circ} \mathrm{C}$. The tissue was mechanically dissociated in DMEM high glucose medium (Sigma) supplemented with $10 \% \mathrm{FCS}, 50 \mathrm{U} / \mathrm{ml}$ penicillin, and 50 $\mathrm{U} / \mathrm{ml}$ streptomycin (DMEM complete), after which the cell suspension was seeded out in T75 culture flasks. Two to three days later, a complete medium change was performed. When $80 \%$ confluency was reached, cells were cultured in DMEM complete supplemented with 30\% LCM. Microglia were obtained 6-7 days later after shake-off $\left(230 \mathrm{rpm}, 3 \mathrm{~h}, 37^{\circ} \mathrm{C}\right)$. Cells were cultured $(0.4$ $\times 10^{6}$ cells $/ \mathrm{ml}$ ) in DMEM complete supplemented with
15\% LCM. For the isolation of CNS-derived leukocytes from EAE animals, the brain and spinal cord were cut into pieces and further homogenized in HBSS supplemented with DNAse. Single-cell suspensions were obtained by pushing the homogenate through a $70 \mu \mathrm{m}$ cell strainer. A discontinuous Percoll gradient (90\%, 60\%, and $40 \%$ ) was used to separate cells from myelin and debris. The cells were gently collected from the 40-60\% interface and washed for further flow cytometric analysis.

\section{Myelin isolation and phagocytosis}

Myelin was purified from postmortem mouse brain tissue by means of density gradient centrifugation, as described previously [37]. Myelin protein concentration was determined using the BCA protein assay kit (Thermo Fisher), according to manufacturer's guidelines. By means of the Chromogenic Limulus Amebocyte Lysate assay kit (Genscript Incorporation), endotoxin content of isolated myelin was determined to be negligible. Cells were treated with $100 \mu \mathrm{g} / \mathrm{ml}$ myelin. To evaluate the ability and extent of myelin phagocytosis, myelin was fluorescently labeled with 1,1'-dioctadecyl-3,3,3',3' -tetramethylindocarbocyanine perchlorate (DiI, Sigma). Cells were exposed to $100 \mu \mathrm{g} / \mathrm{ml}$ DiI-labeled myelin for $1.5 \mathrm{~h}$ and analyzed for fluorescence intensity by using the FACSCalibur (BD Biosciences).

\section{Flow cytometry}

Single-cell suspensions were blocked with $10 \%$ serum and stained with relevant primary antibodies, followed by incubation with the appropriate secondary antibody. To assess lipid load in CNS-derived leukocytes, singlecell suspensions were incubated with Zombie NIR for $15 \mathrm{~min}$, followed by $15 \mathrm{~min}$ incubation with Alexa Fluor 700-labeled anti-CD45. Finally, cells were stained for intracellular lipid load by $15 \mathrm{~min}$ incubation with BODIPY $(493 / 503)$ at $37^{\circ} \mathrm{C}$. To analyze cellular viability, cells were incubated with 7AAD for $10 \mathrm{~min}$. The FACSCalibur or BD LSRFortessa was used to quantify cellular fluorescence.

\section{Quantitative PCR}

Total RNA from cultures was prepared using the RNeasy mini kit (Qiagen), according to manufacturer's instructions. RNA quality was assessed with a NanoDrop spectrophotometer (Isogen Life Science). RNA was converted to cDNA using the reverse transcription system (Quanta Biosciences) and quantitative PCR was subsequently performed on a StepOnePlus detection system (Applied Biosystems). Relative quantification of gene expression was accomplished using the comparative $C_{t}$ method. Data were normalized to the most stable 
reference genes, TATA binding protein $(T b p)$, and cyclin A (Cyca) [38]. Primer sequences are available on request.

\section{Nitrite formation}

To measure the release of NO in supernatants from cell cultures, culture medium was added to an equal volume of $0.05 \% \mathrm{~N}$-(1-Naphthyl)ethylenediamine dihydrochloride, $0.5 \%$ sulfanilamide, and $2.5 \%$ phosphatidic acid solution. Absorbance was measured at $540 \mathrm{~nm}$.

\section{Immunofluorescence}

Murine BMDMs and microglia were cultured on glass cover slides and fixed in 4\% paraformaldehyde for 20 min. Spinal cord tissue of EAE mice was isolated, snapfrozen, and sectioned with a Leica CM1900UV cryostat (Leica Microsystems) to obtain $8 \mu \mathrm{m}$ slices. Frozen human brain material from active MS lesions was obtained from the Netherlands Brain Bank (NBB, Amsterdam, Netherlands). Cryosections were fixed in acetone for 10 min and in 70\% ethanol for 5 min. Immunostaining and analysis of fixed cells and cryosections were performed as described previously [14]. Analysis was carried out using a Nikon eclipse 80i microscope and ImageJ software. Quantification of mean fluorescence intensity (MFI) or percentage positivity was carried out on original images without enhancement. Images shown in figures are digitally enhanced.

\section{Immunohistochemistry}

For 3, 3' diaminobenzidine (DAB) staining, slides were incubated with rat anti-CD36. After washing, HRPconjugated goat anti-rat (Dako) was added. Subsequently, DAB substrate (Dako) was used to stain slides. Intracellular myelin degradation products were visualized with Oil Red O (ORO), which stains neutral lipids. For this purpose, cells and tissue were stained with $0.3 \%$ ORO (Sigma) for $10 \mathrm{~min}$. Cells and cryosections were counterstained with hematoxylin (Merck). Images were acquired using a Leica DM 2000 LED microscope and a Zeiss LSM880 confocal microscope, and quantitative analysis was carried out using the ImageJ software.

\section{Statistical analysis}

Data were statistically analyzed using GraphPad Prism for windows and are reported as mean \pm s.e.m. D'Agostino and Pearson omnibus normality test was used to test normal distribution. An analysis of variances (ANOVA) or two-tailed unpaired student $t$ test (with Welch's correction if necessary) was used for normally distributed data sets. The Kruskal-Wallis or MannWhitney analysis was used for data sets which did not pass normality. $p$ values $<0.05$ were considered to indicate a significant difference $\left(" p<0.05,{ }^{* *} p<0.01\right.$, and $\left.{ }^{* * *} p<0.001\right)$.

\section{Results \\ Myelin uptake increases CD36 abundance in phagocytes in an NRF2-dependent manner}

The internalization of oxLDL, $A \beta$ peptides, and apoptotic cells is well-known to increase CD36 expression in phagocytes [17-26]. To define whether myelin internalization also increases CD36 expression in macrophages and microglia, quantitative PCR, flow cytometry, and immunostaining were used. Our data show that myelin uptake induced mRNA expression of $C d 36$ in murine bone marrow-derived macrophages (BMDMs) and microglia (Fig. 1a). In line with these findings, myelin-treated BMDMs and microglia had elevated protein levels of CD36 (Fig. 1b-d). Also, within demyelinating lesions in the experimental autoimmune encephalomyelitis (EAE) mouse model, foamy IBA1-expressing phagocytes highly expressed CD36 (Fig. 2a). To obtain human validation, active MS lesions that are characterized by the abundant presence of myelin-containing phagocytes were stained for CD36 (Fig. 2b). Immunohistochemical staining demonstrated that CD36 was primarily expressed in highly granular cells resembling myelin-containing phagocytes (Fig. 2b).

Previous studies defined that PPAR $\gamma$ and NRF2 control CD36 expression levels $[34,35]$. By using an antagonist of PPARY (GW9662), we found that myelin did not increase CD36 mRNA expression and protein abundance through PPARy (Fig. 3a and b). In contrast, a marked decrease in CD36 mRNA and protein levels was observed in myelin-treated $\mathrm{Nrf}^{-1-}$ BMDMs as compared to wild-type (wt) BMDMs (Fig. 3c and d). In line with active NRF2 signaling, myelin-treated BMDMs showed an elevated mRNA expression of heme oxygenase 1 (Ho1) and NAD(P)H:quinone acceptor oxidoreductase 1 (Nqo1) (Fig. 3e and f), which was not observed in $\mathrm{Nrf2}^{-1-}$ BMDMs. Myelin internalization did not change the expression of $\mathrm{Nrf2}$ (Fig. 3g), indicating that intracellular myelin accumulation promotes the activation but not expression of NRF2. Collectively, these findings show that myelin increases CD36 expression on gene and protein level in an NRF2-dependent manner.

\section{The NRF2-CD36 signaling axis controls the uptake of myelin by phagocytes}

CD36 is a phagocytic receptor that mediates the uptake of fatty acid-containing ligands [33]. Given the abundance of fatty acids in myelin, we next determined if CD36 controls the uptake of myelin by macrophages and microglia as well. By using sulfo- $\mathrm{N}$-succinimidyl oleate (SSO), a pharmacological CD36 inhibitor (experimental design Fig. 4a), we show that CD36 mediates the 


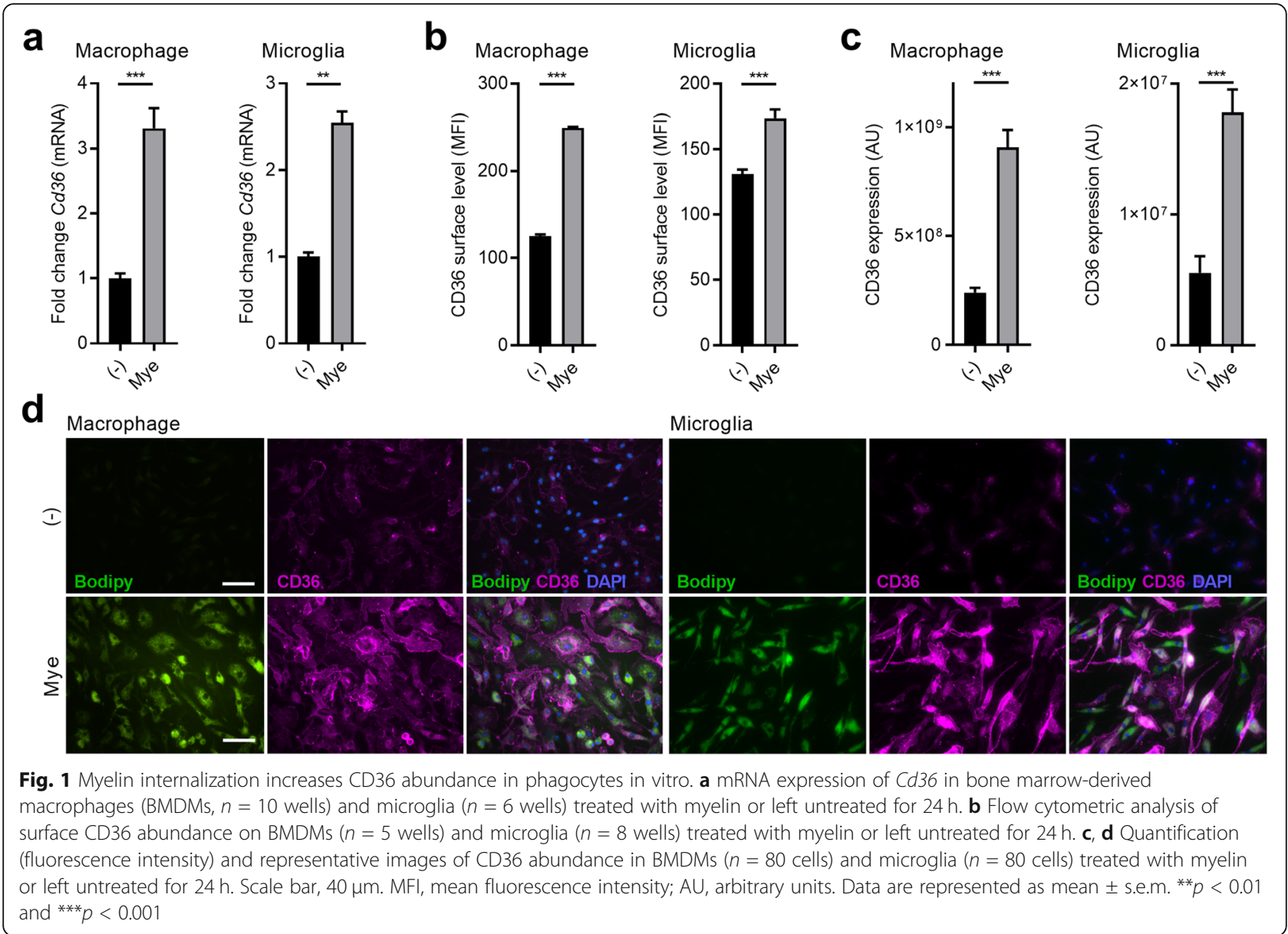

uptake of myelin by control macrophages and those prepretreated with myelin (Fig. 4b), without affecting cell viability (Supplemental Fig. 1). Macrophages pre-treated with myelin showed a reduced phagocytic capacity as compared to control cells, suggesting that myelin internalization activates inhibitory processes that limit the excessive uptake of myelin (Fig. 4b). Similar findings were obtained using primary mouse microglia (Fig. 4b and Supplemental Fig. 1). Notably, CD36 inhibition decreased the phagocytic capacity of macrophages and microglia pre-treated with myelin more prominently than control cells (macrophages, 40\% (control) vs $60 \%$ (myelin pre-treated) reduction; microglia, 34\% (control) vs $75 \%$ (myelin pre-treated) reduction). The latter finding is consistent with the elevated CD36 protein level in these phagocytes following myelin exposure. In line with the reduced phagocytic capacity upon CD36 inhibition, abrogation of CD36 signaling markedly reduced the intracellular lipid load in macrophages upon exposure to myelin (Fig. 4c and d, experimental design Fig. 4a). In summary, these findings indicate that CD36 is involved in the clearance of myelin debris by macrophages and microglia.
Given the importance of NRF2 in controlling CD36 abundance, we next determined if NRF2 underlies the role of CD36 in the clearance of myelin debris. We provide evidence that $\mathrm{Nrf} 2$ deficiency reduces myelin internalization by BMDMs to a similar extent as CD36 inhibition (Fig. 4e). In concordance, neutral lipid levels were significantly decreased in myelin-treated $N r f 2^{-/-}$ BMDMs compared to wt BMDMs (Fig. $4 \mathrm{f}$ and g). Importantly, pharmacological inhibition of CD36 was unable to further decrease the clearance of myelin debris by $N r f 2^{-1-}$ macrophages (Fig. 4h). These findings demonstrate that CD36 controls myelin uptake by macrophages in an NRF2-dependent manner.

\section{CD36-mediated myelin uptake skews macrophages and microglia towards a less-inflammatory phenotype}

Ample evidence indicates that myelin uptake skews phagocytes towards a less-inflammatory phenotype [6-8]. Here, we confirm that intracellular myelin accumulation reduces the inflammatory status of LPS-stimulated macrophages and microglia (experimental design Fig. 5a), evidenced by a reduced mRNA expression of $I l 1 b$, Nos2, Tnfa, Il6, and Ccl5 (Fig. 5b), and a decreased presence of 


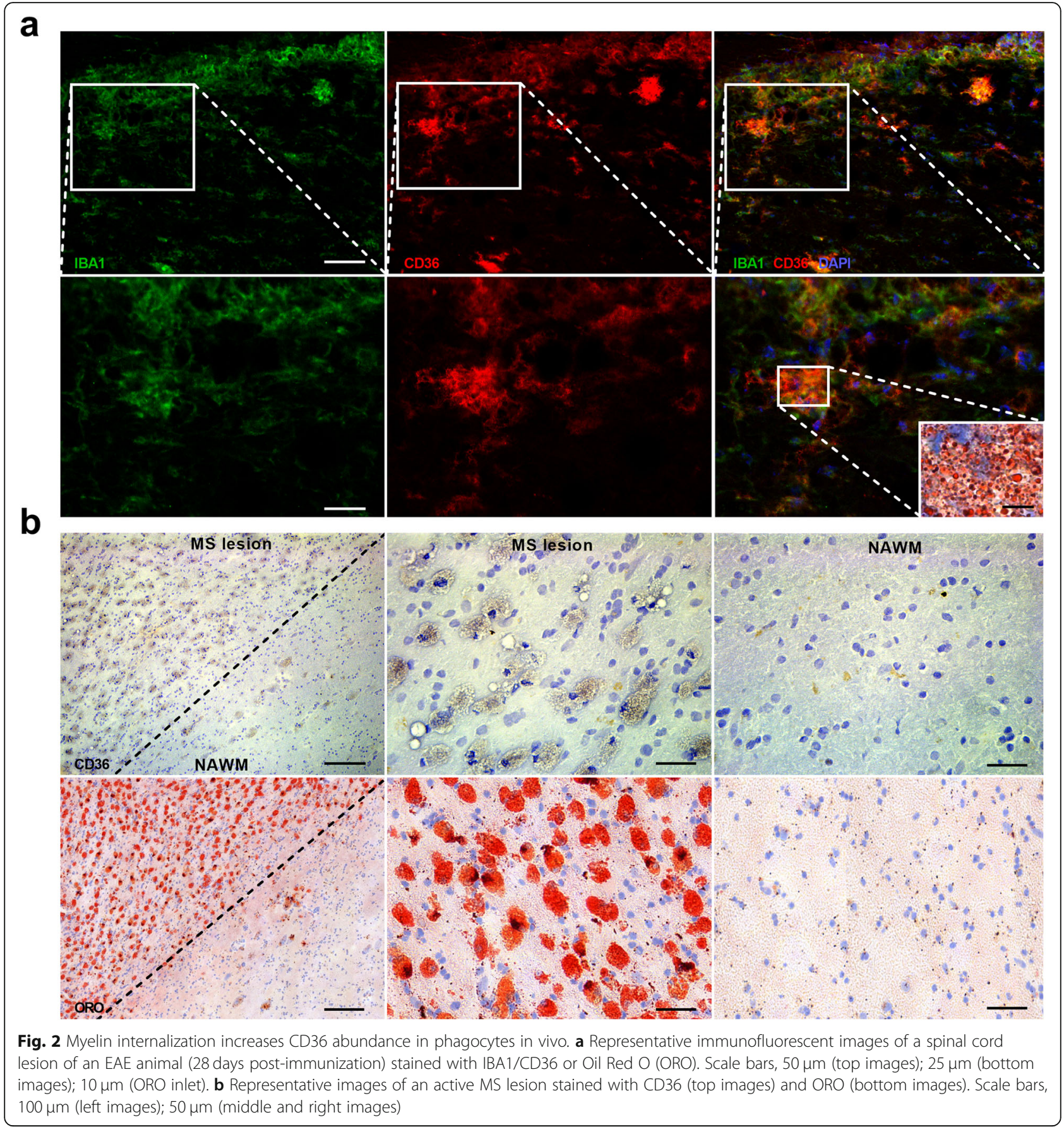

NO in the culture medium (Fig. 5c). Importantly, we show that CD36 inhibition counters the anti-inflammatory impact of myelin on the phenotype of LPS-stimulated as well as unstimulated phagocytes (Fig. 5b and c, Supplemental Fig. 2a). SSO did not impact the inflammatory phenotype of empty macrophages (Supplemental Fig. 2b). Previously, we showed that myelin-mediated activation of LXRs and PPARs underlies the anti-inflammatory impact of myelin on the phenotype of macrophages [9-11]. Consistent with these findings, CD36 inhibition reduced the expression of the LXR- and PPAR-responsive genes Abca1, Scd1, Cpt1a, and Apoe in myelin-treated macrophages (Fig. 5d). Similar findings were observed when using microglia, with the exception of $S c d 1$ expression (Fig. 5d). Interestingly, absence of Nrf2 induced a similar inflammatory and metabolic phenotype in myelin-phagocytosing macrophages (Fig. 5e and f), pointing towards a key role of the NRF2-CD36 signaling axis in driving the phenotype of foamy phagocytes. Collectively, these findings indicate that CD36 inhibition skews myelin-containing macrophages towards an 


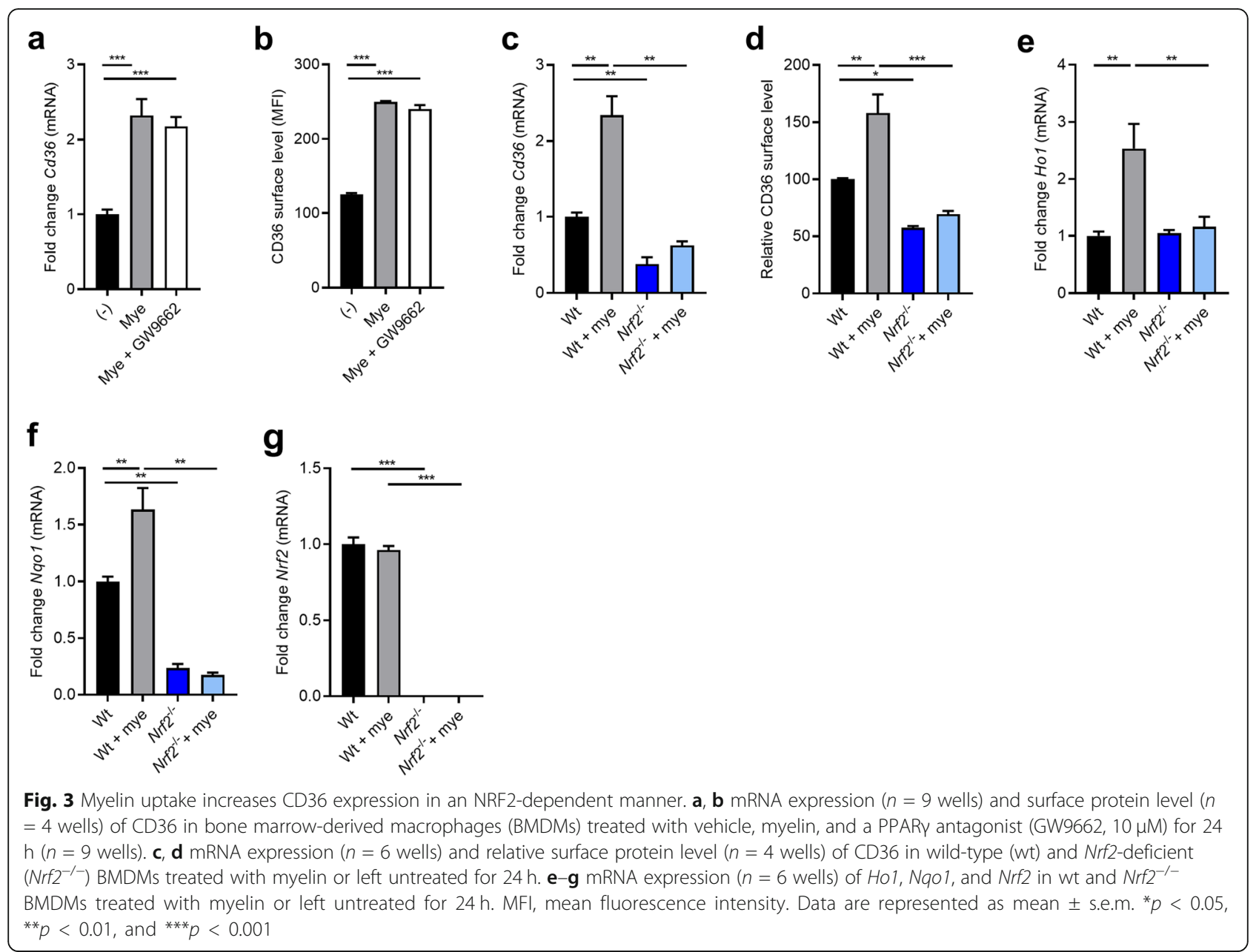

inflammatory phenotype, potentially by reducing the activation of LXRs and PPARs.

\section{CD36 inhibition promotes neuroinflammation in vivo}

Our findings indicate that CD36 inhibition reduces clearance of myelin debris and skews myelin-treated macrophages towards an inflammatory phenotype. To validate our in vitro findings, we next determined the impact of CD36 inhibition on neuroinflammation using the EAE mouse model. Animals treated with the CD36 inhibitor before EAE onset showed a worsened EAE disease severity in the chronic phase compared to vehicletreated animals (Fig. 6a). Immunohistochemical analysis of spinal cord lesions of EAE animals treated with the CD36 inhibitor showed a reduced presence of intracellular $\mathrm{ORO}^{+}$lipid droplets and increased immunoreactivity for degenerated myelin (dMBP) (Fig. 6b-d), with no difference in the number of $\mathrm{F} 4 / 80^{+}$phagocytes (Fig. 6e). Flow cytometric analysis demonstrated that $\mathrm{CD} 45^{+}$leukocytes in the spinal cord of SSO-treated animals had a reduced intracellular lipid load (Fig. 6f). These findings strongly suggest that CD36 inhibition reduces the phagocytosis of myelin debris in vivo. Furthermore, CD36 inhibition increased the neuroinflammatory burden in the spinal cord of EAE animals, as shown by a significantly elevated expression of Nos2, Ccl4, and Ccl5, along with a trend towards increased mRNA levels of Tnfa and Il6 (Fig. 6g). In line with the elevated Nos 2 mRNA expression, treatment with the CD36 inhibitor increased NOS2 reactivity in $\mathrm{F} 4 / 80^{+}$macrophages and microglia within spinal cord lesions (Fig. $6 \mathrm{~h}$ and i). To assess the therapeutic efficacy of CD36 inhibition, EAE animals were treated with SSO after disease onset. In contrast to the prophylactic treatment regime, our findings indicate that SSO treatment does not reduce disease EAE disease severity once clinical symptoms have established (Supplemental Fig. 3). In summary, these findings show that CD36 regulates the uptake of myelin debris by phagocytes in vivo. Furthermore, they suggest that CD36-mediated clearance of myelin debris reduces neuroinflammation by driving phagocytes towards a less-inflammatory phenotype, in particular in early disease stages. 


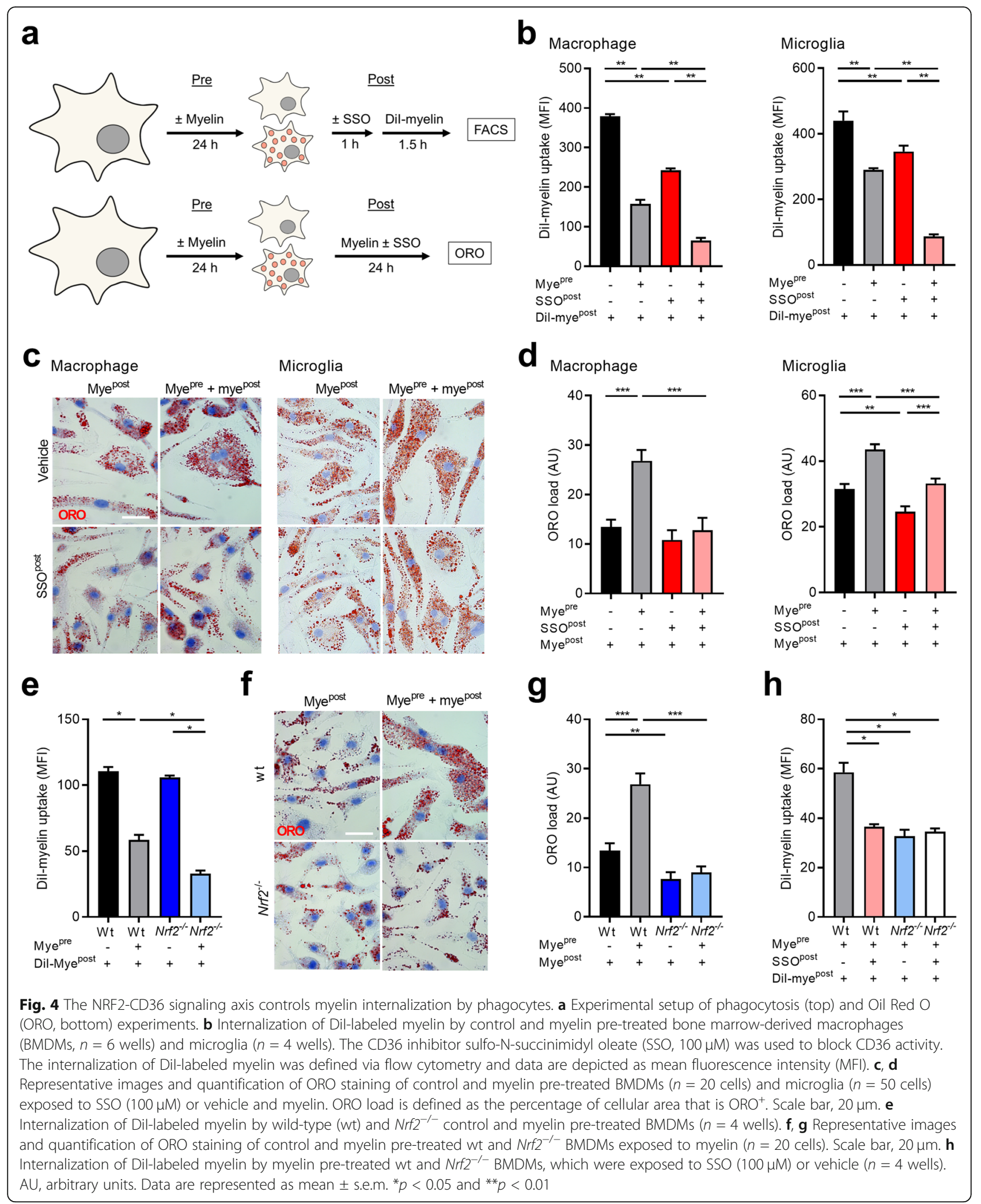




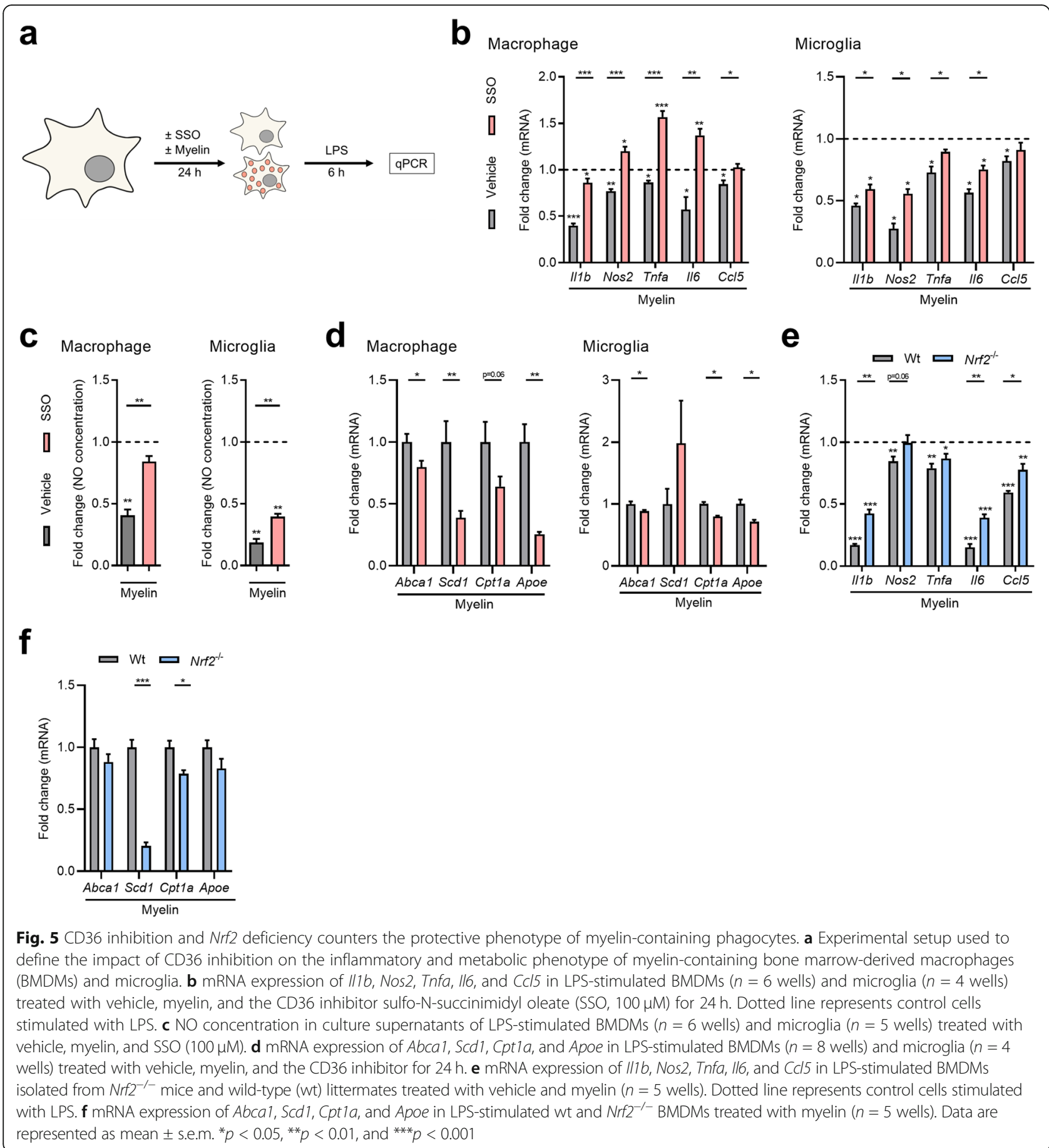

\section{Discussion}

Foamy phagocytes containing myelin remnants are abundantly present in active MS lesions. To date, the receptors that are involved in myelin clearance and their impact on the macrophage phenotype and lesion progression remain to be clarified. Here, we show that fatty acid translocase CD36 controls the uptake of myelin debris by phagocytes and subsequently controls its own expression in an NRF2-dependent manner. Pharmacological inhibition of CD36 countered the initial protective phenotype that phagocytes adopt after myelin uptake, resulting in increased EAE disease severity. Exacerbated neuroinflammation was closely associated with a reduced intracellular phagocytic myelin load and increased presence of myelin debris in the spinal cord lesions. Thus, our findings show that CD36 plays a 


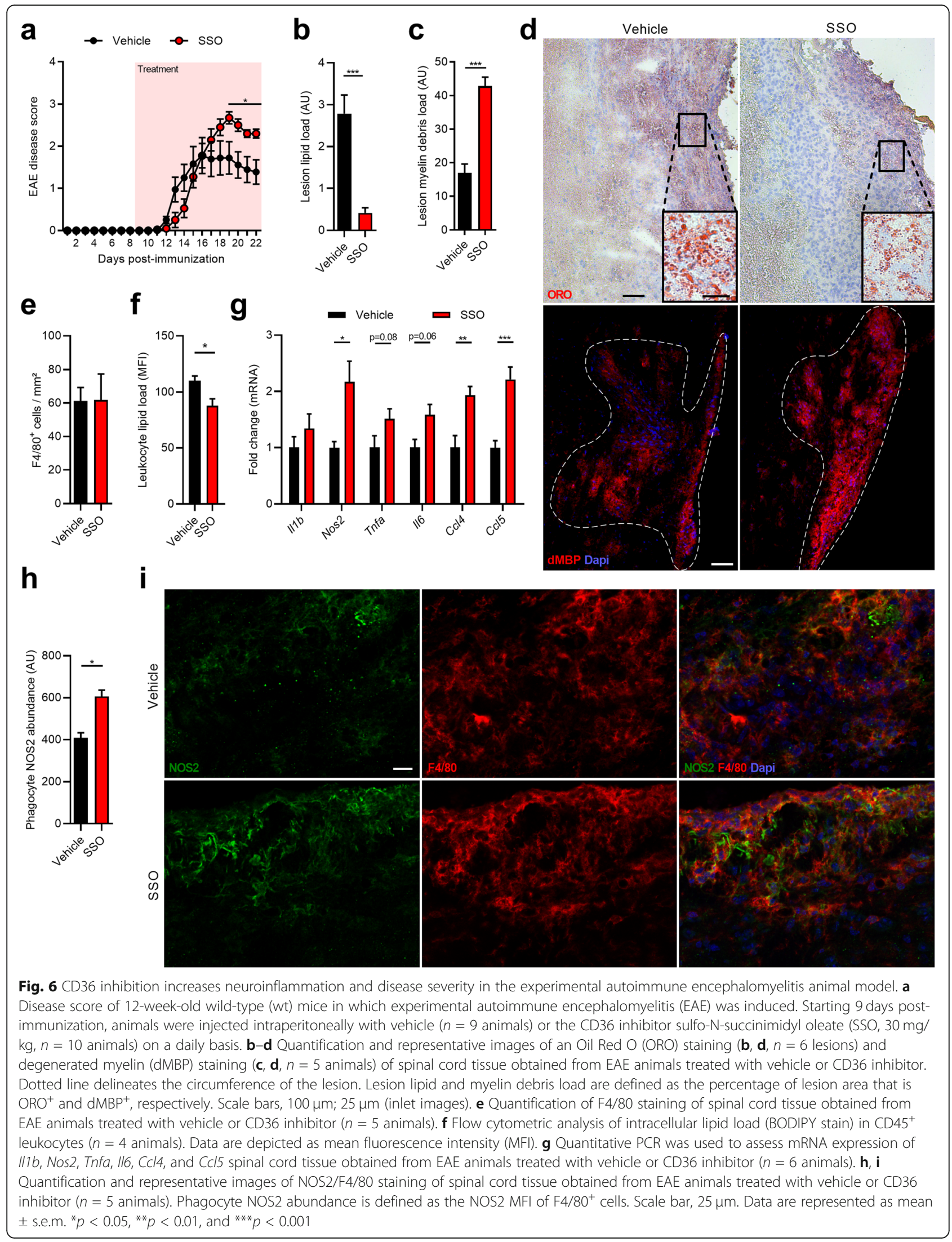


protective role in demyelinating disorders by driving the clearance of myelin debris and suppressing the induction of inflammatory phagocytes.

CD36 is a scavenger receptor involved in the uptake of oxLDL by a mechanism dependent on fatty acid binding [33]. Our findings now indicate that CD36 also mediates the internalization of myelin debris. Given the abundant presence of fatty acids in myelin, it is tempting to speculate that CD36-mediated clearance of myelin depends on fatty acid recognition as well. With respect to the latter, CD36 mediates the uptake of mono- and polyunsaturated long-chain fatty acids such as oleic and docosahexaenoic acid [39, 40], which are highly present in myelin [41]. In future studies, it would be of interest to define the fatty acid substrates in myelin recognized by CD36, and to what extent the levels of these fatty acids are altered in MS. Moreover, as the phagocytosis experiments carried out in this manuscript were performed using myelin debris, future studies should define whether these fatty acid substrates also underlie internalization of intact myelin or whether they are only accessible for CD36 recognition in damaged myelin.

We show that myelin uptake increases CD36 gene expression and protein levels in macrophages and microglia in vitro and within EAE and MS lesions. Correspondingly, CNS lesions in experimental models for ischemic stroke and spinal cord injury (SCI), both characterized by high numbers of myelin-containing cells, show increased levels of CD36 [42, 43]. Interestingly, these findings indicate a positive feedback loop in which CD36-mediated myelin uptake boosts its own expression and secondary uptake of myelin. A similar positive feedback loop has previously been described for CD36 in oxLDL-loaded macrophages in atherosclerotic lesions [44]. Moreover, we recently found that the scavenger receptor CLP1 mediates the uptake of myelin, which subsequently increased its own expression [45]. Collectively, these findings suggest that myelin internalization increases CD36 abundance, thereby sustaining continuous clearance of myelin debris.

Our findings indicate that NRF2 increases CD36 expression in macrophages and microglia upon myelin debris internalization and underlies the impact of CD36 on the phagocytic capacity and inflammatory phenotype of myelin-phagocytosing macrophages. These results are in line with other studies showing the importance of NRF2 in controlling CD36 expression in innate immune cells [35, 46, 47]. The NRF2 pathway is a major regulator of cytoprotective responses to endogenous and exogenous stresses caused by reactive oxygen species (ROS) [48]. On this note, van der Goes et al. demonstrated that myelin internalization triggers the production of ROS, and that scavenging and blocking of ROS reduce the phagocytosis of myelin by macrophages [49]. This study emphasizes the essential role of the oxidative stress response in myelin phagocytosis and provides a rationale for the increased NRF2 activity observed in our cultures. Surprisingly, even though PPAR $\gamma$ controls CD36 expression and myelin-phagocytosing macrophages show active PPAR signaling $[9,34]$, the expression of CD36 in myelin-containing macrophages was not affected by a PPAR $\gamma$ antagonist. Accordingly, we previously demonstrated that PPAR $\gamma$ inhibition does not affect the inflammatory phenotype of myelin-containing macrophages [9]. In summary, our findings highlight the importance of NRF2 in controlling CD36 abundance in macrophages upon myelin internalization.

Previously, we and others showed that myelin uptake induces a less-inflammatory, reparative phenotype via the activation of the lipid-sensing nuclear receptors LXRs and PPARs $[9,10]$. Here, we report that pharmacological inhibition of CD36 activity prevents the induction of the less-inflammatory phenotype of macrophages in vitro, and in parallel, reduces the expression of LXRand PPAR-responsive genes in these cells. A reduced uptake of myelin-derived cholesterol and fatty acids and a consequent decreased LXR and PPAR activity might well explain the inflammatory phenotype of macrophages upon CD36 inhibition in vitro.

Ample evidence indicates that macrophages and microglia have both disease-promoting and resolving functions in demyelinating CNS disorders [1]. Given the inhibitory role of myelin debris on oligodendrocyte maturation, the clearance of damaged myelin debris by phagocytes is considered to be essential for CNS repair $[5,50]$. Here, we show that CD36 inhibition increases disease severity at the chronic disease stage of EAE when treatment started before disease onset. Aggravated disease severity was associated with a reduced phagocyte myelin load, increased presence of non-cell associated degenerated myelin, and elevated neuroinflammatory burden in the CNS. These findings strongly suggest that CD36 inhibition reduces the clearance of myelin debris by phagocytes and promotes their inflammatory phenotype in vivo. In line with our findings, Zhu et al. showed that $C d 36$ deficiency in the SCI mouse model also reduced lesional phagocyte lipid content. However, in this study absence of Cd36 improved lesion size and locomotor recovery [42]. One possible explanation for this ambiguity is the difference in lesional phagocytic lipid load between these models. In the EAE model, the extent of demyelination is relatively limited compared to the SCI model. On this note, we showed that the intracellular myelin load determines the inflammatory phenotype of macrophages and microglia [13]. It is tempting to speculate that blocking myelin uptake in the SCI model is beneficial because the induction of inflammatory features associated with an elevated intracellular 
myelin overload is prevented. Notably, our findings further indicate that that CD36 inhibition does not affect EAE disease severity once administrated after disease onset. The extensive amount of demyelination and thus phagocytic myelin uptake in the early, non-symptomatic disease stage might well explain why CD36 inhibition had no effect in this experimental setup [51, 52]. More research is necessary to characterize the underlying mechanism of the beneficial and harmful effects of myelin clearance in different diseases and model systems, and to define the optimal timing of CD36 inhibitor administration.

\section{Conclusion}

Our study provides the first evidence for an essential role of CD36 in the uptake of myelin and in controlling the inflammatory properties of phagocytes in demyelinating disorders. By mediating myelin debris clearance, CD36 induces a protective phenotype in myelin-laden macrophages and microglia, and dampens EAE disease severity. Hence, targeting CD36 holds therapeutic promise for demyelinating disorders such as MS.

\section{Supplementary information}

Supplementary information accompanies this paper at https://doi.org/10. 1186/s12974-020-01899-x.

Additional file 1: Supplemental Figure S1. CD36 inhibition does not affect phagocyte viability. Percentage of viable bone marrow-derived macrophages (BMDMs, $n=6$ wells) and microglia ( $n=4$ wells) after treatment with vehicle or the CD36 inhibitor sulfo-N-succinimidyl oleate $(\mathrm{SSO}, 100 \mu \mathrm{M})$ for $24 \mathrm{~h}$. Data are represented as mean \pm s.e.m.

Additional file 2: Supplemental Figure S2. CD36 inhibition modestly increases the pro-inflammatory phenotype of naive myelin-treated macrophages. a mRNA expression of 1116 , Nos2, Tnfa, 116 , and C 15 in bone marrow-derived macrophages (BMDMs, $n=6$ wells) treated with vehicle, myelin, and the CD36 inhibitor sulfo-N-succinimidyl oleate (SSO, $100 \mu \mathrm{M}$ ) for $24 \mathrm{~h}$. Dotted line represents control cells. b mRNA expression of $1 / 1 b$ Nos2, Tnfa, 116 , and Ccl5 in BMDMs ( $n=6$ wells) treated with vehicle or SSO $(100 \mu \mathrm{M})$. Data are represented as mean \pm s.e.m. ${ }^{*} p<0.05$ and ** $p<0.01$.

Additional file 3: Supplemental Figure S3. CD36 inhibition has no effect on experimental autoimmune encephalomyelitis disease progression when treatment starts after disease onset. Disease score of 12-week-old wild-type (wt) mice in which experimental autoimmune encephalomyelitis (EAE) was induced. When a disease score of 0.5 or higher was obtained, animals were injected intraperitoneally with vehicle $(n=7$ animals) or the CD36 inhibitor sulfo-N-succinimidyl oleate (SSO, $30 \mathrm{mg}$ / $\mathrm{kg}, n=8$ animals) on a daily basis. Data are represented as mean \pm s.e.m.

\section{Abbreviations}

7AAD: 7-Aminoactinomycin D; BMDMs: Bone marrow-derived macrophages; CLP1: Collectin placenta 1; CNS: Central nervous system; Dil: 1,1'-dioctadecyl3,3,3',3'-tetramethylindocarbocyanine perchlorate; dMBP: Degenerated myelin basic protein; EAE: Experimental autoimmune encephalomyelitis; HO1: Heme oxygenase 1; LPS: Lipopolysaccharide; LXR: Liver X receptor; MFI: Mean fluorescence intensity; MS: Multiple sclerosis; NO: Nitrite; NRF2: Nuclear factor erythroid 2-related factor 2; NQO1: NAD(P)H:quinone acceptor oxidoreductase 1; ORO : Oil red O; ROS: Reactive oxygen species; SCl: Spinal cord injury; SSO: Sulfo-N-succinimidyl oleate; PPAR: Peroxisome proliferatoractivated receptor
}

\section{Acknowledgements}

The authors thank Marie-Paule Tulleners for excellent technical assistance.

\section{Authors' contributions}

$\mathrm{EG}, \mathrm{MH}, \mathrm{JH}$, and JB conceived experiments. EG, EW, BvdH, MH, TD, JVB, CE, and JB performed experiments. EG, EW, BvdH, MH, KH, TD, JVB, CE, SH, SK-R, JH, and $J B$ discussed results. KH and SK-R contributed reagents and materials. EG and JB wrote the manuscript. EG, EW, BvdH, MH, KH, TD, JVB, CE, SH, SK-R, JH, and JB revised the manuscript. The author(s) read and approved the final manuscript.

\section{Funding}

The work has been supported by the Flemish Fund for Scientific Research (FWO Vlaanderen; 12J9116N, 12JG119N, 12U7718N, and G099618N), the Belgian Charcot Foundation (FCS-2016-EG7, R-8676, and R-6832), and the special research fund UHasselt (BOF).

\section{Availability of data and materials}

The datasets used and/or analyzed during the current study are available through the corresponding author on reasonable request.

\section{Ethics approval and consent to participate}

Brain tissue samples were obtained from the Netherlands Brain Bank (coordinator Dr. Huitinga, Amsterdam, The Netherlands). The Netherlands Brain Bank received permission to perform autopsies for the use of tissue and for access to medical records for research purposes from the Ethical Committee of the VU University Medical Center, Amsterdam, The Netherlands. All patients and controls, or their next of kin, had given informed consent for autopsy and use of brain tissue for research purposes. All experimental protocols using human tissue were conducted in accordance with institutional guidelines and approved by the Medical Ethical Committee of Hasselt University.

\section{Consent for publication}

Not applicable.

\section{Competing interests}

The authors declare that they have no competing interests.

\section{Author details}

${ }^{1}$ Department of Immunology and Infection, Biomedical Research Institute, Hasselt University, Diepenbeek, Belgium. ${ }^{2}$ Inflammation, Microbiome and Immunosurveillance, INSERM UMR99, Université Paris-Saclay,

Châtenay-Malabry, France.

Received: 12 March 2020 Accepted: 15 July 2020

Published online: 27 July 2020

\section{References}

1. Grajchen E, Hendriks JJA, Bogie JFJ. The physiology of foamy phagocytes in multiple sclerosis. Acta Neuropathol Commun. 2018;6:124.

2. Bogie JF, Stinissen P, Hendriks JJ. Macrophage subsets and microglia in multiple sclerosis. Acta Neuropathol. 2014;128:191-213.

3. Holmoy T, Hestvik AL. Multiple sclerosis: immunopathogenesis and controversies in defining the cause. Curr Opin Infect Dis. 2008;21:271-8.

4. Lampron A, Larochelle A, Laflamme N, Prefontaine P, Plante MM, Sanchez MG, Yong WW, Stys PK, Tremblay ME, Rivest S. Inefficient clearance of myelin debris by microglia impairs remyelinating processes. J Exp Med. 2015;212: 481-95

5. Ruckh JM, Zhao JW, Shadrach JL, van Wijngaarden P, Rao TN, Wagers AJ, Franklin RJ. Rejuvenation of regeneration in the aging central nervous system. Cell Stem Cell. 2012;10:96-103.

6. Bogie JF, Stinissen P, Hellings N, Hendriks JJ. Myelin-phagocytosing macrophages modulate autoreactive T cell proliferation. J Neuroinflammation. 2011;8:85.

7. Boven LA, Van Meurs M, Van Zwam M, Wierenga-Wolf A, Hintzen RQ, Boot RG, Aerts JM, Amor S, Nieuwenhuis EE, Laman JD. Myelin-laden macrophages are anti-inflammatory, consistent with foam cells in multiple sclerosis. Brain. 2006;129:517-26.

8. Hikawa N, Takenaka T. Myelin-stimulated macrophages release neurotrophic factors for adult dorsal root ganglion neurons in culture. Cell Mol Neurobiol. $1996 ; 16: 517-28$. 
9. Bogie JF, Jorissen W, Mailleux J, Nijland PG, Zelcer N, Vanmierlo T, Van Horssen J, Stinissen P, Hellings N, JJA H. Myelin alters the inflammatory phenotype of macrophages by activating PPARs. Acta Neuropathologica Communications. 2013:1

10. Bogie JF, Timmermans S, Huynh-Thu VA, Irrthum A, Smeets HJ, Gustafsson JA, Steffensen KR, Mulder M, Stinissen P, Hellings N, Hendriks JJ. Myelinderived lipids modulate macrophage activity by liver $X$ receptor activation. PLoS One. 2012;7:e44998.

11. Mailleux J, Vanmierlo T, Bogie JF, Wouters E, Lutjohann D, Hendriks JJ, van Horssen J. Active liver $X$ receptor signaling in phagocytes in multiple sclerosis lesions. Mult Scler. 2018;24:279-89.

12. Cantuti-Castelvetri L, Fitzner D, Bosch-Queralt M, Weil MT, Su M, Sen P, Ruhwedel T, Mitkovski M, Trendelenburg G, Lutjohann D, et al. Defective cholesterol clearance limits remyelination in the aged central nervous system. Science. 2018;359:684-8.

13. Bogie JFJ, Grajchen E, Wouters E, Corrales AG, Dierckx T, Vanherle S, Mailleux J, Gervois P, Wolfs E, Dehairs J, et al. Stearoyl-CoA desaturase-1 impairs the reparative properties of macrophages and microglia in the brain. J Exp Med. 2020;217.

14. Bogie JF, Mailleux J, Wouters E, Jorissen W, Grajchen E, Vanmol J, Wouters K, Hellings N, Van Horsen J, Vanmierlo T, Hendriks JJ. Scavenger receptor collectin placenta 1 is a novel receptor involved in the uptake of myelin by phagocytes. Sci Rep. 2017;7:44794.

15. Healy LM, Perron G, Won SY, Michell-Robinson MA, Rezk A, Ludwin SK, Moore CS, Hall JA, Bar-Or A, Antel JP. MerTK is a functional regulator of myelin phagocytosis by human myeloid cells. J Immunol. 2016;196:3375-84.

16. Gaultier A, Wu X, Le Moan N, Takimoto S, Mukandala G, Akassoglou K, Campana WM, Gonias SL. Low-density lipoprotein receptor-related protein 1 is an essential receptor for myelin phagocytosis. J Cell Sci. 2009;122:115562

17. Ohtani K, Suzuki Y, Eda S, Kawai T, Kase T, Keshi H, Sakai Y, Fukuoh A, Sakamoto T, Itabe H, et al. The membrane-type collectin CL-P1 is a scavenger receptor on vascular endothelial cells. J Biol Chem. 2001;276:44222-8.

18. Costales P, Fuentes-Prior P, Castellano J, Revuelta-Lopez E, Corral-Rodriquez MA, Nasarre L, Badimon L, Llorente-Cortes V. K domain CR9 of low density lipoprotein $(\mathrm{LDL})$ receptor-related protein $1(\mathrm{LRP} 1)$ is critical for aggregated $\mathrm{LDL}$-induced foam cell formation from human vascular smooth muscle cells. J Biol Chem. 2015;290:14852-65.

19. Steinbrecher UP. Receptors for oxidized low density lipoprotein. Biochim Biophys Acta. 1999;1436:279-98.

20. Nakamura K, Ohya W, Funakoshi H, Sakaguchi G, Kato A, Takeda M, Kudo T, Nakamura T. Possible role of scavenger receptor SRCL in the clearance of amyloid-beta in Alzheimer's disease. J Neurosci Res. 2006;84:874-90.

21. Savage JC, Jay T, Goduni E, Quigley C, Mariani MM, Malm T, Ransohoff RM, Lamb BT, Landreth GE. Nuclear receptors license phagocytosis by trem2+ myeloid cells in mouse models of Alzheimer's disease. J Neurosci. 2015;35: 6532-43.

22. Yamada K, Hashimoto T, Yabuki C, Nagae Y, Tachikawa M, Strickland DK, Liu Q, Bu G, Basak JM, Holtzman DM, et al. The low density lipoprotein receptor-related protein 1 mediates uptake of amyloid beta peptides in an in vitro model of the blood-brain barrier cells. J Biol Chem. 2008;283:34554-62.

23. Lai AY, MCLaurin J. Clearance of amyloid-beta peptides by microglia and macrophages: the issue of what, when and where. Future Neurol. 2012;7: $165-76$

24. Scott RS, McMahon EJ, Pop SM, Reap EA, Caricchio R, Cohen PL, Earp HS, Matsushima GK. Phagocytosis and clearance of apoptotic cells is mediated by MER. Nature. 2001;411:207-11.

25. Nilsson A, Vesterlund L, Oldenborg PA. Macrophage expression of LRP1, a receptor for apoptotic cells and unopsonized erythrocytes, can be regulated by glucocorticoids. Biochem Biophys Res Commun. 2012;417:1304-9.

26. Gordon S, Pluddemann A. Macrophage clearance of apoptotic cells: a critical assessment. Front Immunol. 2018:9:127.

27. Pepino MY, Kuda O, Samovski D, Abumrad NA. Structure-function of CD36 and importance of fatty acid signal transduction in fat metabolism. Annu Rev Nutr. 2014;34:281-303.

28. Coburn CT, Knapp FF Jr, Febbraio M, Beets AL, Silverstein RL, Abumrad NA Defective uptake and utilization of long chain fatty acids in muscle and adipose tissues of CD36 knockout mice. J Biol Chem. 2000;275:32523-9.

29. Hames KC, Vella A, Kemp BJ, Jensen MD. Free fatty acid uptake in humans with CD36 deficiency. Diabetes. 2014;63:3606-14.
30. Febbraio M, Podrez EA, Smith JD, Hajjar DP, Hazen SL, Hoff HF, Sharma K, Silverstein RL. Targeted disruption of the class B scavenger receptor CD36 protects against atherosclerotic lesion development in mice. J Clin Invest. 2000;105:1049-56.

31. Febbraio M, Guy E, Silverstein RL. Stem cell transplantation reveals that absence of macrophage CD36 is protective against atherosclerosis. Arterioscler Thromb Vasc Biol. 2004;24:2333-8.

32. Guy E, Kuchibhotla S, Silverstein R, Febbraio M. Continued inhibition of atherosclerotic lesion development in long term Western diet fed CD360 /apoEo mice. Atherosclerosis. 2007;192:123-30.

33. Jay AG, Chen AN, Paz MA, Hung JP, Hamilton JA. CD36 binds oxidized low density lipoprotein (LDL) in a mechanism dependent upon fatty acid binding. J Biol Chem. 2015;290:4590-603.

34. Zhou J, Febbraio M, Wada T, Zhai Y, Kuruba R, He J, Lee JH, Khadem S, Ren S, $\mathrm{Li}$, et al. Hepatic fatty acid transporter Cd36 is a common target of LXR, PXR, and PPARgamma in promoting steatosis. Gastroenterology. 2008;134:556-67.

35. Helou DG, Noel B, Gaudin F, Groux H, El Ali Z, Pallardy M, Chollet-Martin S, Kerdine-Romer S. Cutting edge: Nrf2 regulates neutrophil recruitment and accumulation in skin during contact hypersensitivity. J Immunol. 2019;202: 2189-94.

36. Itoh $\mathrm{K}$, Chiba $\mathrm{T}$, Takahashi $\mathrm{S}$, Ishii $\mathrm{T}$, Igarashi $\mathrm{K}$, Katoh $\mathrm{Y}$, Oyake $\mathrm{T}$, Hayashi $\mathrm{N}$ Satoh K, Hatayama I, et al. An Nrf2/small Maf heterodimer mediates the induction of phase II detoxifying enzyme genes through antioxidant response elements. Biochem Biophys Res Commun. 1997;236:313-22.

37. Mailleux J, Timmermans S, Nelissen K, Vanmol J, Vanmierlo T, van Horssen J, Bogie JFJ, Hendriks JJA. Low-density lipoprotein receptor deficiency attenuates neuroinflammation through the induction of apolipoprotein $\mathrm{E}$. Front Immunol. 2017:8:1701

38. Vandesompele J, De Preter K, Pattyn F, Poppe B, Van Roy N, De Paepe A, Speleman F. Accurate normalization of real-time quantitative RT-PCR data by geometric averaging of multiple internal control genes. Genome Biol. 2002;3 RESEARCH0034.

39. Pohl J, Ring A, Korkmaz U, Ehehalt R, Stremmel W. FAT/CD36-mediated long-chain fatty acid uptake in adipocytes requires plasma membrane rafts. Mol Biol Cell. 2005;16:24-31.

40. Takai M, Kozai Y, Tsuzuki S, Matsuno Y, Fujioka M, Kamei K, Inagaki H, Equchi A, Matsumura S, Inoue K, Fushiki T. Unsaturated long-chain fatty acids inhibit the binding of oxidized low-density lipoproteins to a model CD36. Biosci Biotechnol Biochem. 2014;78:238-44.

41. Chrast R, Saher G, Nave KA, Verheijen MH. Lipid metabolism in myelinating glial cells: lessons from human inherited disorders and mouse models. J Lipid Res. 2011;52:419-34.

42. Zhu Y, Lyapichev K, Lee DH, Motti D, Ferraro NM, Zhang Y, Yahn S, Soderblom C, Zha J, Bethea JR, et al. Macrophage transcriptional profile identifies lipid catabolic pathways that can be therapeutically targeted after spinal cord injury. J Neurosci. 2017;37:2362-76.

43. Cho S, Park EM, Febbraio M, Anrather J, Park L, Racchumi G, Silverstein RL, ladecola $C$. The class B scavenger receptor CD36 mediates free radical production and tissue injury in cerebral ischemia. J Neurosci. 2005;25:2504-12.

44. Nakata A, Nakagawa Y, Nishida M, Nozaki S, Miyagawa J, Nakagawa T, Tamura R, Matsumoto K, Kameda-Takemura K, Yamashita S, Matsuzawa Y. CD36, a novel receptor for oxidized low-density lipoproteins, is highly expressed on lipid-laden macrophages in human atherosclerotic aorta. Arterioscler Thromb Vasc Biol. 1999;19:1333-9.

45. Bogie JFJ, Mailleux J, Wouters E, Jorissen W, Grajchen E, Vanmol J, Wouters K, Hellings N, Horssen JV, Vanmierlo T, Hendriks JJA. Corrigendum: scavenger receptor collectin placenta 1 is a novel receptor involved in the uptake of myelin by phagocytes. Sci Rep. 2017;7:46925.

46. Ishii T, Itoh K, Ruiz E, Leake DS, Unoki H, Yamamoto M, Mann GE. Role of $\mathrm{Nrf2}$ in the regulation of CD36 and stress protein expression in murine macrophages: activation by oxidatively modified LDL and 4hydroxynonenal. Circ Res. 2004;94:609-16.

47. Aubouy A, Olagnier D, Bertin G, Ezinmegnon S, Majorel C, Mimar S, Massougbodji A, Deloron P, Pipy B, Coste A. Nrf2-driven CD36 and HO-1 gene expression in circulating monocytes correlates with favourable clinical outcome in pregnancy-associated malaria. Malar J. 2015;14:358.

48. Olagnier D, Lavergne RA, Meunier E, Lefevre L, Dardenne C, Aubouy A, Benoit-Vical F, Ryffel B, Coste A, Berry A, Pipy B. Nrf2, a PPARgamma alternative pathway to promote CD36 expression on inflammatory macrophages: implication for malaria. PLoS Pathog. 2011;7:e1002254. 
49. van der Goes A, Brouwer J, Hoekstra K, Roos D, van den Berg TK, Dijkstra $C D$. Reactive oxygen species are required for the phagocytosis of myelin by macrophages. J Neuroimmunol. 1998;92:67-75.

50. Miron VE, Boyd A, Zhao JW, Yuen TJ, Ruckh JM, Shadrach JL, van

Wijngaarden P, Wagers AJ, Williams A, Franklin RJ, French-Constant C. M2 microglia and macrophages drive oligodendrocyte differentiation during CNS remyelination. Nat Neurosci. 2013.

51. Yamasaki R, Lu H, Butovsky O, Ohno N, Rietsch AM, Cialic R, Wu PM, Doykan CE, Lin J, Cotleur AC, et al. Differential roles of microglia and monocytes in the inflamed central nervous system. J Exp Med. 2014;211:1533-49.

52. Fu Y, Frederick TJ, Huff TB, Goings GE, Miller SD, Cheng JX. Paranodal myelin retraction in relapsing experimental autoimmune encephalomyelitis visualized by coherent anti-Stokes Raman scattering microscopy. J Biomed Opt. 2011;16:106006.

\section{Publisher's Note}

Springer Nature remains neutral with regard to jurisdictional claims in published maps and institutional affiliations.

Ready to submit your research? Choose BMC and benefit from:

- fast, convenient online submission

- thorough peer review by experienced researchers in your field

- rapid publication on acceptance

- support for research data, including large and complex data types

- gold Open Access which fosters wider collaboration and increased citations

- maximum visibility for your research: over $100 \mathrm{M}$ website views per year

At $\mathrm{BMC}$, research is always in progress.

Learn more biomedcentral.com/submissions 